\title{
Targeted Genomic Sequencing Reveals Novel TP53 In-frame Deletion Mutations Leading to p53 Overexpression in High-grade Serous Tubo-ovarian Carcinoma
}

\author{
YOON YANG JUNG ${ }^{1}$, HA YOUNG WOO ${ }^{2}$ and HYUN-SOO KIM ${ }^{3}$ \\ ${ }^{1}$ Department of Pathology, Myongji Hospital, Hanyang University College of Medicine, Goyang, Republic of Korea; \\ ${ }^{2}$ Department of Pathology, Severance Hospital, Yonsei University College of Medicine, Seoul, Republic of Korea; \\ ${ }^{3}$ Department of Pathology and Translational Genomics, Samsung Medical Center, \\ Sungkyunkwan University School of Medicine, Seoul, Republic of Korea
}

\begin{abstract}
Background/Aim: High-grade serous carcinoma (HGSC) is the most common histological subtype of ovarian carcinoma. Somatic mutation of tumor protein 53 (TP53) is a hallmark of tubo-ovarian HGSC and is observed in almost all such cases. Highly sensitive targeted genomic sequencing can be used to identify novel mutations that may become potential druggable targets and aid in therapeutic decisions. The aim of this study was to describe the clinicopathological and molecular characteristics of HGSCs with novel somatic TP53 mutations identified by next-generation sequencing (NGS). Materials and Methods: A commercial NGS panel comprising 170 genes, including TP53, was used to analyze the genetic profiles of 132 ovarian carcinoma cases. The clinicopathological characteristics and p53 immunostaining results of two HGSCs exhibiting novel TP53 mutations were investigated. Results: Eighty-eight (66.7\%) out of 132 ovarian carcinoma cases were diagnosed as HGSC. Novel TP53 inframe deletion mutations c.719_727delGTTCCTGCA (p53 p.Ser240_Cys242del) and c.634_642delTTTCGACAT (p53 p.F212_H214del) were detected in a single case of HGSC each. Both patients were postmenopausal women. Imaging and laboratory studies revealed peritoneal carcinomatosis and elevated levels of serum tumor markers. The patients underwent primary debulking surgery and were diagnosed as having stage IIIC HGSC. In both cases, p53 immunostaining revealed uniform nuclear immunoreactivity in $90 \%$ or more of tumor cells at a very strong intensity. Conclusion: Targeted
\end{abstract}

Correspondence to: Hyun-Soo Kim, Department of Pathology and Translational Genomics, Samsung Medical Center, Sungkyunkwan University School of Medicine, 81, Irwon-ro, Gangnam-gu, Seoul 06351, Republic of Korea. Tel: +82 234101243, Fax: +82 234100025, e-mail: hyun-soo.kim@samsung.com

Key Words: Ovary, high-grade serous carcinoma, TP53 in-frame deletion, p53 overexpression. genomic sequencing revealed novel in-frame deletion mutations of TP53 leading to p53 overexpression in tuboovarian HGSC. This discovery of previously unreported somatic TP53 mutations provides insight into the translation of NGS technology into personalized medicine and identifies new potential targets for therapeutic applications.

Tumor protein 53 (TP53) encodes the transcription factor p53, which promotes the transcription of genes involved in cellcycle arrest, cellular senescence, apoptosis, metabolism, DNA repair, and other processes following cellular stress $(1,2)$. Loss of p53 function is a common feature in human malignancies, and TP53 mutation is a major cause of such loss (3), occurring in more than half of all human carcinomas (4). In some carcinomas, TP53 mutation is an independent marker of poor prognosis (5). Studies on the p53 pathway and its interaction networks are promising sources of insight for the discovery of therapeutic targets for TP53-mutated malignancies (6). Some therapeutic strategies have been proposed to treat TP53mutated carcinomas, such as the restoration of wild-type p53 activity, promotion of mutant p53 degradation, or targeting of pathways regulated by mutant p53 (7).

TP53 mutations include single-base substitutions leading to missense or nonsense mutations, in-frame insertions or deletions (indels), and frameshift indels, as well as mutations that affect splice sites (8). While other tumor-suppressor genes, such as retinoblastoma $(R B)$ and breast cancer 1 , early onset $(B R C A l)$, are commonly inactivated by frameshift or nonsense mutations, missense mutations are the predominant type of TP53 mutation (3). These mutations occur primarily in exons 4-9, which encode the DNA-binding domain of the p53 protein (9). Tumor cells with missense TP53 mutations produce full-length p53 proteins containing only a single amino acid substitution. The resulting mutant p53 proteins exhibit prolonged half-lives and accumulate within tumor cell nuclei. Diffuse and strong nuclear p53 immunoreactivity is regarded as indicative of TP53 missense mutation $(10,11)$. 
Table I. List of genes sequenced in this study using next-generation sequencing.

\begin{tabular}{|c|c|c|c|c|c|c|}
\hline$A B L 1$ & $B R C A 2$ & DNMT3A & FOXL2 & MCL1 & NTRK2 & RSPO2 \\
\hline$A B L 2$ & $B R D 2$ & DOT1L & GNA11 & $M D M 2$ & NTRK3 & RUNX1 \\
\hline$A K T 1$ & $B R D 3$ & $E G F R$ & GNAQ & MDM4 & NUTM1 & $S M A D 2$ \\
\hline$A K T 2$ & $B R D 4$ & ЕРНA3 & GNAS & MED12 & $P D G F B$ & SMAD4 \\
\hline AKT3 & $C B F B$ & $E R B B 2$ & HDAC9 & MEN1 & PDGFRA & SMARCA \\
\hline$A L K$ & $C C N D 1$ & ERBB3 & $H G F$ & $M E T$ & PDGFRB & $S M A R C B$ \\
\hline$A P C$ & $C C N D 2$ & ERBB 4 & $H R A S$ & $M I T F$ & $P I K 3 C A$ & $S M O$ \\
\hline$A R$ & $C C N D 3$ & ERCC2 & $I D H 1$ & $M L H 1$ & PIK $3 C B$ & $S R C$ \\
\hline$A R A F$ & CCNE1 & $E R G$ & $I D H 2$ & $M P L$ & $P I K 3 C D$ & STK11 \\
\hline$A S X L 1$ & $\mathrm{CDH1}$ & ERRFII & $I G F 1 R$ & MSH2 & PIK3R1 & $S Y K$ \\
\hline$A T M$ & CDK12 & ESR1 & $I G F 2$ & MSH6 & PIK3R2 & $T E R T$ \\
\hline ATR & CDK4 & ETV1 & $J A K 1$ & $M T O R$ & POLE & TET2 \\
\hline AURKA & CDK6 & ETV4 & $J A K 2$ & $M Y C$ & PPARG & TMPRSS2 \\
\hline$A U R K B$ & $C D K N 1 A$ & ETV5 & $J A K 3$ & $M Y C N$ & PTCH1 & TOP $2 A$ \\
\hline$A U R K C$ & $C D K N 1 B$ & ETV6 & $K D R$ & MYD88 & PTEN & TP53 \\
\hline$A X L$ & $C D K N 2 A$ & EWSR1 & KIT & $N F 1$ & $R A B 35$ & TSC1 \\
\hline$B A P 1$ & $C D K N 2 B$ & $E Z H 2$ & $K M T 2 A$ & $N F 2$ & RAD50 & TSC2 \\
\hline$B C L 2$ & $C D K N 2 C$ & $F B X W 7$ & KRAS & NFKBIA & $R A F 1$ & $V H L$ \\
\hline$B R A F$ & CEBPA & $F G F R 1$ & $M A P 2 K 1$ & NKX2-1 & $R A R A$ & $W T 1$ \\
\hline BRCAl & CHEK2 & FGFR2 & $M A P 2 K 2$ & NOTCH1 & $R B 1$ & XPO1 \\
\hline$B R C A 2$ & $C R E B B P$ & FGFR3 & $M A P 2 K 4$ & NOTCH2 & $R E T$ & ZNRF3 \\
\hline$B R D 2$ & $C R K L$ & FGFR4 & MAP $3 K 1$ & NOTCH3 & RHEB & \\
\hline$B R D 3$ & CSF1R & $F L C N$ & $M A P 3 K 4$ & NOTCH4 & RICTOR & \\
\hline$B R D 4$ & $C T N N B 1$ & FLT1 & MAPK1 & NPM1 & $R N F 43$ & \\
\hline$B R A F$ & $D D R 1$ & FLT3 & МAPK3 & $N R A S$ & ROS1 & \\
\hline$B R C A 1$ & $D D R 2$ & FLT4 & МАРK 8 & NTRK1 & RSPOI & \\
\hline
\end{tabular}

In contrast, TP53 nonsense mutation leads to the formation of a truncated, non-immunoreactive protein and the complete absence of p53 expression (12-14).

Ovarian carcinoma is the leading cause of death among patients with gynecological malignancies. More than $75 \%$ of patients are diagnosed with disease in advanced stages (15, 16) and have a 5-year survival rate of approximately $25 \%(8$, 17). Despite advances in surgical techniques, chemotherapy, and imaging modalities, more than $60 \%$ of patients experience relapse in the first 3 years owing to the development of chemotherapeutic resistance (16). High-grade serous carcinoma (HGSC) is the most common histological subtype of ovarian carcinoma, accounting for up to $70 \%$ of all ovarian carcinoma cases. It has recently been suggested that all HGSCs are in fact characterized by TP53 mutation (18).

Over the past few years, next-generation sequencing (NGS) technology has been widely implemented to characterize the genetic profiles of patients, as it exhibits improved sensitivity in mutation detection, faster turnaround times, and reduced costs compared to traditional sequencing methods $(16,19)$. In this study on patients with HGSC, we performed targeted sequencing of HGSC tumor tissues using a commercial NGS gene panel. In cases with novel TP53 mutations, clinicopathological characteristics were explored, and impacts on 553 protein expression were evaluated by immunohistochemical staining.

\section{Materials and Methods}

Case selection. Following approval (4-2017-0993) by the Institutional Review Board of Severance Hospital, 132 ovarian carcinoma cases were initially collected. In cases with novel TP53 mutations, clinicopathological characteristics including patient age, clinical presentation, imaging findings, serum tumor marker levels, final pathological diagnosis, histological grade, International Federation of Gynecology and Obstetrics (FIGO) stage, architecture, nuclear pleomorphism, mitotic activity, atypical mitotic figure, coagulative tumor cell necrosis, intratumoral hemorrhage, and immunostaining results were obtained from the electronic medical records and pathology reports.

Pathological examination. Resected tissues were initially examined by pathologists before fixation in $10 \%$ neutral-buffered formalin. After fixation, the tissues were thoroughly examined macroscopically and sectioned. Sections were processed with an automatic tissue processor (Peloris II; Leica Microsystems, Newcastle upon Tyne, UK) and embedded in paraffin blocks. Slices 4 - $\mu$ m-thick were cut from each formalin-fixed, paraffin-embedded (FFPE) tissue block and stained with hematoxylin and eosin. Two Board-certified pathologists specialized in gynecological oncology examined all available hematoxylin and eosin-stained slides by light microscopy and made pathological diagnoses. The most representative slide for each case was chosen for immunostaining and targeted sequencing.

Targeted sequencing. Genomic DNA was extracted using a QIAamp DNA FFPE Tissue Kit (Qiagen, Valencia, CA, USA). Using genomic DNA, the coding exons and their flanking regions of 171 genes (Table 
Table II. Clinicopathological characteristics of patients with high-grade serous carcinoma with novel in-frame deletion mutations of tumor protein 53 (TP53).

\begin{tabular}{lcc}
\hline Characteristic & Patient 1 & Patient 2 \\
\hline Age (years) & 56 & 52 \\
Clinical presentation & Abdominal distension and constipation & Abdominal pain \\
Imaging finding & & \\
MRI & Bilateral ovarian masses with peritoneal seeding & \\
PET-CT & Increased FDG uptake in the bilateral ovarian \\
& masses and abdominopelvic peritoneum & Diffuse peritoneal thickening \\
Serum tumor marker level & & Increased FDG uptake in the \\
CA 125 & $1,739.6$ Ubdominopelvic peritoneum \\
HE4 & 354.5 pmol/1 & $2,535.6$ U/ml \\
Diagnosis & High-grade serous carcinoma & 453.0 pmol/1 \\
Histological grade & 3 & High-grade serous carcinoma \\
FIGO stage & IIIC & 3 \\
Dominant architectural pattern & Papillary & IIIC \\
Nuclear pleomorphism & Present & Papillary \\
Mitotic activity & Present & Present \\
Atypical mitotic figure & Present & Present \\
Coagulative tumor cell necrosis & Present & Present \\
Intratumoral hemorrhage & Present & Present \\
p53 immunostaining result & Diffuse and strongly positive & Present
\end{tabular}

CA 125: Cancer antigen 125; FDG: fluorodeoxyglucose; FIGO: International Federation of Gynecology and Obstetrics; HE4: human epididymis protein 4; MRI: magnetic resonance imaging; PET-CT: positron-emission tomography-computed tomography.

I) were enriched using the SureSelectXT Reagent Kit (Agilent Technologies, Santa Clara, CA, USA) (20-22). The products were sequenced on a HiSeq 2500 System (Illumina, San Diego, CA, USA) using paired-end reads. The reads were aligned to the reference genome (Genome Reference Consortium Human Build 37) obtained from the University of California Santa Cruz Genome Browser database (https://genome.ucsc.edu/) (23), and duplicate reads were removed. The Burrows-Wheeler Aligner (http://bio-bwa.sourceforge.net/), SAMtools (http://samtools.sourceforge.net/), Picard Tools (http://broadinstitute. github.io/picard/), and Genome Analysis Toolkit (https://software. broadinstitute.org/gatk/) were used for sorting Sequence Alignment Map/Binary Alignment Map files, duplicate marking, and local realignment. Local realignment and base recalibration were carried out using the Single Nucleotide Polymorphism Database (https://www.ncbi. nlm.nih.gov/projects/SNP/), Mills indel reference (24), HapMap (https://www.ncbi.nlm.nih.gov/probe/docs/projhapmap/), and Omni (http://www.internationalgenome.org/category/omni/). Single-nucleotide variants and insertions/deletions were identified using MuTect (http://archive.broadinstitute.org/cancer/cga/mutect) and Pindel (http:// gmt.genome.wustl.edu/packages/pindel/), respectively. ANNOVAR (http://annovar.openbioinformatics.org/) was used to annotate the detected variants. Any single-nucleotide variant present at $>0.1 \%$ in the Exome Variant Server (https://evs.gs.washington.edu/) or Single Nucleotide Polymorphism Database was filtered. Variants present in the Catalogue of Somatic Mutations in Cancer (https://cancer. sanger.ac.uk/) were reviewed.

Immunostaining. Immunostaining was performed using an automatic instrument (Ventana Benchmark XT; Ventana Medical Systems, Tucson, AZ, USA) according to the manufacturer's recommendations $(11,20-22,25-29)$. Antigen retrieval was performed using Cell Conditioning Solution (CC1; Ventana
Medical Systems). The 4- $\mu$ m-thick FFPE slices were incubated with anti-p53 (1:300, clone DO-7; Novocastra, Newcastle upon Tyne, UK). After chromogenic visualization using an ultraView Universal DAB Detection Kit (Ventana Medical Systems), slices were counterstained with hematoxylin. Appropriate positive and negative controls were concurrently stained to validate the staining method. The negative control was prepared by substituting nonimmune serum for primary antibody, resulting in no detectable staining. The p53 immunostaining patterns were interpreted as missense mutation, nonsense mutation, or wild-type when p53 expression was diffuse and strong ( $>60 \%$ of tumor cell nuclei), completely absent $(0 \%)$, or focal and weakly positive, respectively $(11,20,26)$.

\section{Results}

Baseline characteristics. From January 2016 to December 2018, we obtained tumor tissue samples from 132 patients with ovarian carcinoma for targeted sequencing analysis. Eighty-eight $(66.7 \%)$ cases were diagnosed as HGSC, 16 $(12.1 \%)$ as clear-cell carcinoma, $13(9.8 \%)$ as endometrioid carcinoma, six $(4.5 \%)$ as mucinous carcinoma, five $(3.8 \%)$ as seromucinous carcinoma, and four (3.0\%) as carcinosarcoma. The ages of these 88 patients with HGSC ranged from 36 to 81 (median $=60$ years; mean $=57.7$ years). All patients underwent debulking surgery, consisting of total hysterectomy, bilateral salpingo-oophorectomy, pelvic and para-aortic lymph node dissection, total omentectomy, peritonectomy, with/without appendectomy. 
At least one genomic alteration was identified in all 132 patients, with TP53 mutation being the most frequent. All but three HGSC cases $(85 / 88 ; 96.6 \%)$ exhibited TP53 mutations. BRCA1 or breast cancer 2, DNA repairassociated (BRCA2) mutation was also detected in 10 (11.4\%) and five $(5.7 \%)$ HGSC cases, respectively. In contrast, clear-cell carcinoma, endometrioid carcinoma, and seromucinous carcinoma cases were found to have mutations in phosphatidylinositol-4,5-bisphosphate 3kinase catalytic subunit alpha $(P I K 3 C A)$, phosphoinositide3-kinase regulatory subunit $1(P I K 3 R 1)$, AT-rich interactive domain-containing protein $1 \mathrm{~A}(A R I D I A)$, phosphatase and tensin homolog deleted on chromosome 10 (PTEN), and catenin $\beta 1$ (CTNNB1). Three cases of mucinous carcinoma exhibited v-Ki-ras2 Kirsten rat sarcoma viral oncogene homolog $(K R A S)$ mutation, while one exhibited B-Raf proto-oncogene, serine/threonine kinase $(B R A F)$ mutation. Some cases exhibited amplification of cyclin E1 (CCNE1) or ERB-B2 receptor tyrosine kinase 2 (ERBB2). Other mutations were classified as variants of uncertain significance (30).

In addition to missense and nonsense mutations and frameshift indels, some unusual TP53 mutations were detected in the HGSC cases. Three cases had splice site mutations, and two had in-frame deletion mutations. Since we previously investigated HGSC cases with TP53 splice site mutations (11), we focused in this study on the two cases of HGSC with TP53 in-frame deletion mutations.

Clinicopathological characteristics of tubo-ovarian HGSC with TP53 in-frame deletion mutation. Table II summarizes the clinicopathological features of two patients with HGSC with TP53 in-frame deletion mutations. Patient 1, a 56-yearold Korean woman, presented with abdominal distension and constipation. Magnetic resonance imaging revealed bilateral ovarian masses and peritoneal seeding. Positron-emission tomography-computed tomography (PET-CT) revealed increased fluorodeoxyglucose (FDG) uptake in the bilateral ovarian masses and abdominopelvic peritoneum. Serum levels of cancer antigen 125 (CA-125) and human epididymis protein 4 (HE4) were elevated $(1,739.6 \mathrm{U} / \mathrm{mI}$ and $354.5 \mathrm{pmol} / \mathrm{I}$, respectively). The clinical impression was bilateral ovarian carcinoma with peritoneal carcinomatosis. She was diagnosed as having grade 3 ovarian HGSC after primary debulking surgery including total hysterectomy, bilateral salpingo-oophorectomy, pelvic and para-aortic lymph node dissection, total omentectomy, appendectomy, and peritonectomy. The FIGO stage was IIIC.

Patient 2, a 52-year-old Korean woman, presented with abdominal pain. Magnetic resonance imaging revealed diffuse thickening of the abdominopelvic peritoneum. PETCT revealed increased FDG uptake in the abdominopelvic peritoneum. Elevated serum levels of CA-125 (2,535.6
$\mathrm{U} / \mathrm{mI}$ ) and HE4 (453.0 pmol/I) were observed. Based on the clinical impression of primary peritoneal carcinomatosis, she underwent primary debulking surgery, consisting of total hysterectomy, bilateral salpingo-oophorectomy, pelvic and para-aortic lymph node dissection, total omentectomy, and peritonectomy. She was diagnosed as having grade 3 ovarian HGSC, with a FIGO stage of IIIC.

Histopathologically, both cases showed characteristic morphological features of HGSC. The tumor tissue samples of patient 1 showed destructive infiltration of tumor cells forming branching papillary fronds, slit-like fenestrations, and a complex glandular architecture (Figure 1A). The individual tumor cells showed high-grade nuclear atypia. Similarly, in the tissue samples of patient 2, the tumor cells displayed severe nuclear pleomorphism, frequent mitoses, and atypical mitotic figures (Figure 1B), as well as forming a papillary and solid architecture.

NGS results. Table III summarizes the sequencing results for the two HGSCs with TP53 in-frame deletion mutations. The identified variants were automatically annotated by comparison against the human TP53 reference genomic sequence NC_000017.10 (chr $17: 7,571,720-7,590,868)$ corresponding to isoform NM_000546.5. In patient 1, c.719_727delGTTCCTGCA, encompassing the deletion of nine nucleotides in exon 7 of TP53, was identified with an allelic frequency of $35.01 \%$. This is an in-frame deletion mutation that causes the deletion of the second and third nucleotides in codon 240 (A $\underline{\text { GT}}$; deleted nucleotides are underlined), encoding a serine, and the seven subsequent nucleotides, including the complete codons 241 (Ser) and 242 (Cys) and the first nucleotide in codon 243 (ATG), encoding methionine. Thus, the protein-coding frame is preserved, with the translation of a methionine residue resulting from the remaining nucleotides (ATG) in codons 240 and 243, but with the removal of three amino acid residues (Ser240, Ser241, Cys242) in the DNA-binding domain of the mature p53 protein (p53 p.Ser240_Cys242del).

In patient 2, c.634_642delTTTCGACAT, encompassing the deletion of nine nucleotides in exon 6 of TP53, was identified with an allelic frequency of $13.02 \%$. This is another in-frame deletion mutation that leads to the deletion of three amino acids: Phe212, Arg213, and His214 (p53 p.F212_H214del).

Immunostaining results. In the HGSC samples of patient 1, a very high percentage (almost $100 \%$ ) of tumor cell nuclei were positive for p53 immunoreactivity, and a very strong intensity was observed (Figure 1C). The tumor tissue samples of patient 2 also exhibited uniform nuclear p53 immunoreactivity in up to $90 \%$ of tumor cells with very strong intensity (Figure 1D). 


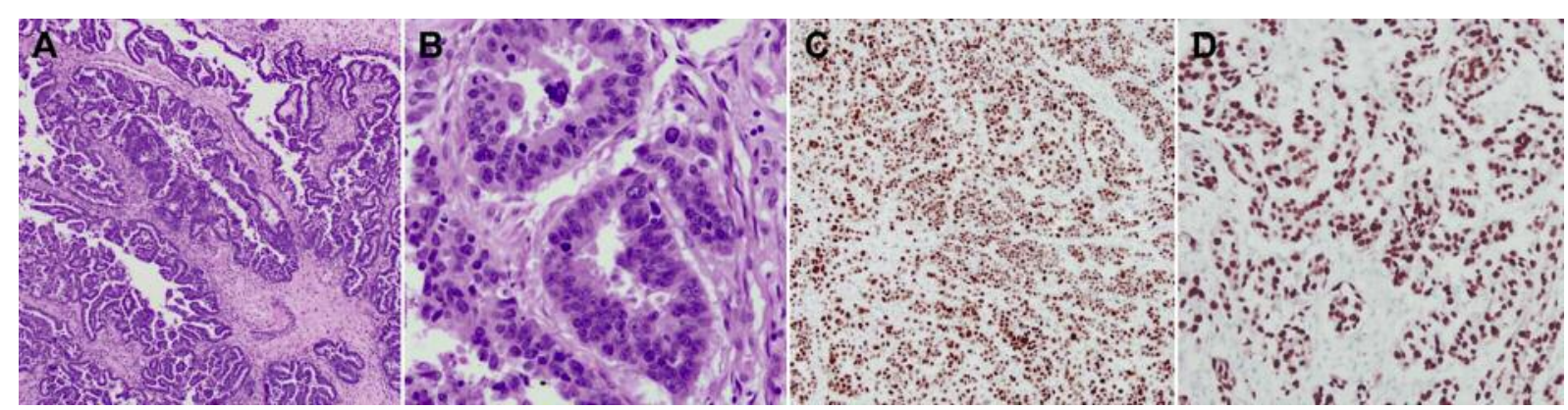

Figure 1. Histopathological findings $(A$ and $B)$ and p53 immunostaining results $(C$ and $D)$ in patients with high-grade serous carcinoma with novel in-frame deletion mutations of tumor protein 53 (TP53). A: Case 1: At low-power magnification (40x), the tumor cells showed destructive infiltration and formed a papillary architecture, slit-like fenestrations, and complex glands with intraluminal necrotic debris. B: Case 2: High-power magnification (400x) showed severe nuclear pleomorphism, conspicuous nucleoli, frequent mitoses, and atypical mitotic figures. C: Case 1: Mediumpower magnification (100x) revealed tumor cells to be diffusely and strongly positive for p53 protein (usually characteristic of a missense mutation pattern). D: Case 2: High-power magnification (200x) showed cells displayed diffuse and strong nuclear p53 immunoreactivity.

Table III. Targeted sequencing results from patients with high-grade serous carcinoma with novel in-frame deletion mutations of tumor protein 53 (TP53).

\begin{tabular}{|c|c|c|c|c|c|c|c|}
\hline $\begin{array}{l}\text { Patient } \\
\text { no. }\end{array}$ & Sample & $\begin{array}{l}\text { Gene } \\
\text { type }\end{array}$ & $\begin{array}{l}\text { Mutation } \\
\text { type }\end{array}$ & $\begin{array}{l}\text { Sequence } \\
\text { change }\end{array}$ & $\begin{array}{c}\text { p53 } \\
\text { domain }\end{array}$ & $\begin{array}{c}\text { Variant frequency } \\
\text { allele }(\%)\end{array}$ & $\begin{array}{l}\text { Amino acid } \\
\text { change }\end{array}$ \\
\hline 1 & FFPE (PDS) & $\begin{array}{c}\text { TP53 } \\
\text { CDKN2A }\end{array}$ & $\begin{array}{l}\text { In-frame deletion } \\
\text { Missense mutation }\end{array}$ & $\begin{array}{c}\text { c.719_727delGTTCCTGCA } \\
\text { c. } 428 \mathrm{C}>\mathrm{G}\end{array}$ & DBD & $\begin{array}{l}35.01 \\
81.44\end{array}$ & $\begin{array}{c}\text { p.S240_C242del } \\
\text { p.A143G }\end{array}$ \\
\hline 2 & FFPE (PDS) & $\begin{array}{c}\text { TP53 } \\
\text { SRC } \\
\text { ARID1A }\end{array}$ & $\begin{array}{l}\text { In-frame deletion } \\
\text { Missense mutation } \\
\text { Frameshift insertion }\end{array}$ & $\begin{array}{c}\text { c.634_642delTTTCGACAT } \\
\text { c.548C }>\text { T } \\
\text { c.862_863insCGCCCCC }\end{array}$ & DBD & $\begin{array}{c}13.62 \\
48.31 \\
5.48\end{array}$ & $\begin{array}{l}\text { p.F212_H214del } \\
\text { p.T183M } \\
\text { p.Q288Pfs*114 }\end{array}$ \\
\hline
\end{tabular}

ARID1A: AT-rich interactive domain-containing protein 1A; CDKN2A: cyclin-dependent kinase Inhibitor 2A; DBD: DNA-binding domain; del: deletion; FFPE: formalin-fixed, paraffin-embedded tissue; ins: insertion; PDS: primary debulking surgery; SRC: v-src avian sarcoma (SchmidtRuppin A-2) viral oncogene homolog; VUS: variant of uncertain significance.

\section{Discussion}

The massively parallel sequencing capability of NGS technology has enabled the simultaneous analysis of genomic aberrations in multiple genes in a single assay. Recently, NGS technology has become more affordable, leading to large, collaborative studies on whole genomes and the identification of target genes and predictive biomarkers in various malignancies. In the Republic of Korea, since March 2017, NGS gene panel testing of patients with ovarian carcinoma has been included in the National Health Insurance Service, and the number of NGS tests has consequently increased.

The p53 monomer contains 393 amino acids and is encoded by 11 exons comprising different functional domains, the most relevant of which are the central sitespecific DNA-binding domain, the oligomerization domain, and a strongly basic C-terminal domain $(16,31)$. Although the spectrum of TP53 mutations is highly heterogeneous, with the detection of in-frame or frameshift indels, non-sense mutations, and splice site mutations, missense mutations in the protein-coding sequence leading to amino acid changes are the most common (32). Approximately $90 \%$ of known TP53 variants, mostly of the missense type, cluster between exons 4 and 9, encoding the highly conserved DNA-binding domain, with mutations being particularly common at specific hot-spot codons $(16,33)$.

TP53 mutation, accompanied by aberrant p53 expression, is the main molecular feature of tubo-ovarian HGSC. Yemelyanova et al. showed that p53 immunostaining results, including a combination of p53 overexpression (>60\%) and the complete absence of p53 expression, correlated with TP53 mutational status in $94.4 \%$ of HGSC cases $(11,34)$. Another study using similar cut-off values $(>70 \%$ for overexpression and $<5 \%$ for lack of expression) showed matching results between immunostaining and mutational analyses in $95.8 \%$ of HGSC cases (8). Previous studies have demonstrated that diffuse and strong p53 expression is indicative of a missense TP53 mutation $(10,11,35,36)$. However, in this study, diffuse and strong nuclear p53 immunoreactivity was observed in two HGSC cases with novel TP53 in-frame deletion mutations. 
Both these mutations are located in the region encoding the DNA-binding domain, which is the functional core of p53 and is directly involved in its interaction with DNA; thus, they may produce slightly truncated p53 isoforms, resulting in marked nuclear p53 overexpression (37). Structural integrity and DNAbinding domain folding are required for wild-type p53 to bind to its target genes and function as a transcription factor. The proper DNA-binding domain conformation is also required for nuclear export of p53 following ubiquitination $(38,39)$. These mutations may induce conformational changes, leading to impaired mutant p53 degradation, as evidenced by the strong nuclear p53 accumulation observed.

The two patients described herein were diagnosed with FIGO stage IIIC HGSC. Their imaging and laboratory findings, as well as histopathological features, were typical of HGSC. Compared to the clinicopathological features of HGSCs with missense or nonsense TP53 mutation, the features of the cases with in-frame deletion mutations were not obviously different. The clinical relevance and pathogenetic implications of the novel mutations remain uncertain without conclusive data from functional studies. A previous study reported that it is difficult to draw conclusions regarding TP53 in-frame deletion mutations, even when a loss-of-function effect can be excluded via immunostaining (16). p53 binds DNA as a tetramer, and it is well accepted that inactivation of wild-type p53 through dominant-negative effects requires tetramerization $(40,41)$. Mutant alleles in TP53 may lead to the formation of mixed heterotetramers and confer unknown dominant-negative effects on the remaining wild-type p53 alleles.

In conclusion, we report the detection of novel somatic TP53 mutations in two patients with HGSC using an NGS approach. The functional impact and prognostic value of these novel in-frame deletion mutations in TP53 remain to be elucidated. Further studies are warranted to determine whether these mutations may be potential therapeutic targets for personalized treatment. It is clear that the best approach for targeting mutant p53 will depend on the type of mutation and its functional impact. The discovery of previously unreported TP53 mutations indicates the potential for translating NGS results into personalized treatment by identifying new potential druggable targets for future therapeutic applications. Our findings also improve our knowledge of the complex genomic heterogeneity of ovarian carcinoma. Future directions will be to define the clinical value of somatic TP53 mutations in a large cohort of patients with advanced ovarian carcinoma, particularly HGSC.

\section{Acknowledgements}

This research was supported by the faculty grant of Myongji Hospital (1801-06-03) and the Basic Science Research Program through the National Research Foundation of Korea (NRF), funded by the Ministry of Education (2016R1D1A1B03935584).

\section{References}

1 Kastenhuber ER and Lowe SW: Putting p53 in context. Cell 170: 1062-1078, 2017. PMID: 28886379. DOI: 10.1016/ j.cell.2017.08.028

2 Ungerleider NA, Rao SG, Shahbandi A, Yee D, Niu T, Frey WD and Jackson JG: Breast cancer survival predicted by TP53 mutation status differs markedly depending on treatment. Breast Cancer Res 20: 115, 2018. PMID: 30285883. DOI: 10.1186/ s13058-018-1044-5

3 Kang HJ, Chun SM, Kim KR, Sohn I and Sung CO: Clinical relevance of gain-of-function mutations of p53 in high-grade serous ovarian carcinoma. PLoS One 8: e72609, 2013. PMID: 23967324. DOI: 10.1371/journal.pone.0072609

4 Hollstein M, Sidransky D, Vogelstein B and Harris CC: p53 mutations in human cancers. Science 253: 49-53, 1991. PMID: 1905840.

5 Petitjean A, Achatz MI, Borresen-Dale AL, Hainaut P and Olivier M: TP53 mutations in human cancers: Functional selection and impact on cancer prognosis and outcomes. Oncogene 26: 21572165, 2007. PMID: 17401424. DOI: 10.1038/sj.onc.1210302

6 Khoo KH, Verma CS and Lane DP: Drugging the p53 pathway: Understanding the route to clinical efficacy. Nat Rev Drug Discov 13: 217-236, 2014. PMID: 24577402. DOI: 10.1038/nrd4236

7 Wang X and Sun Q: TP53 mutations, expression and interaction networks in human cancers. Oncotarget 8: 624-643, 2017. PMID: 27880943. DOI: 10.18632/oncotarget.13483

8 Cole AJ, Dwight T, Gill AJ, Dickson KA, Zhu Y, Clarkson A, Gard GB, Maidens J, Valmadre S, Clifton-Bligh R and Marsh DJ: Assessing mutant p53 in primary high-grade serous ovarian cancer using immunohistochemistry and massively parallel sequencing. Sci Rep 6: 26191, 2016. PMID: 27189670. DOI: 10.1038/srep26191

9 Rivlin N, Brosh R, Oren M and Rotter V: Mutations in the p53 tumor-suppressor gene: Important milestones at the various steps of tumorigenesis. Genes Cancer 2: 466-474, 2011. PMID: 21779514. DOI: $10.1177 / 1947601911408889$

10 Bennett WP, Hollstein MC, Hsu IC, Sidransky D, Lane DP, Vogelstein B and Harris CC: Mutational spectra and immunohistochemical analyses of p53 in human cancers. Chest 101: 19S-20S, 1992. PMID: 1541189.

$11 \mathrm{Na} \mathrm{K}$, Sung JY and Kim HS: TP53 mutation status of tuboovarian and peritoneal high-grade serous carcinoma with a wildtype p53 immunostaining pattern. Anticancer Res 37: 66976703, 2017. PMID: 29187446. DOI: 10.21873/anticanres.12128

12 Lax SF, Kendall B, Tashiro H, Slebos RJ and Hedrick L: The frequency of $p 53, K$-ras mutations, and microsatellite instability differs in uterine endometrioid and serous carcinoma: evidence of distinct molecular genetic pathways. Cancer 88: 814-824, 2000. PMID: 10679651

13 Shahin MS, Hughes JH, Sood AK and Buller RE: The prognostic significance of $p 53$ tumor-suppressor gene alterations in ovarian carcinoma. Cancer 89: 2006-2017, 2000. PMID: 11064359.

14 Tashiro H, Isacson C, Levine R, Kurman RJ, Cho KR and Hedrick L: p53 gene mutations are common in uterine serous carcinoma and occur early in their pathogenesis. Am J Pathol 150: 177-185, 1997. PMID: 9006334.

15 Hennessy BT, Coleman RL and Markman M: Ovarian cancer. Lancet 374: 1371-1382, 2009. PMID: 19793610. DOI: 10.1016/ S0140-6736(09)61338-6

16 Garziera M, Cecchin E, Canzonieri V, Sorio R, Giorda G, Scalone S, De Mattia E, Roncato R, Gagno S, Poletto E, Romanato L, 
Sartor F, Polesel $\mathbf{J}$ and Toffoli G: Identification of novel somatic TP53 mutations in patients with high-grade serous ovarian cancer (HGSOC) using next-generation sequencing (NGS). Int J Mol Sci 19: 1510, 2018. PMID: 29783665. DOI: 10.3390/ijms 19051510

17 Visintin I, Feng Z, Longton G, Ward DC, Alvero AB, Lai Y, Tenthorey J, Leiser A, Flores-Saaib R, Yu H, Azori M, Rutherford T, Schwartz PE and Mor G: Diagnostic markers for early detection of ovarian cancer. Clin Cancer Res 14: 1065-1072, 2008. PMID: 18258665. DOI: 10.1158/1078-0432.CCR-07-1569

18 Vang R, Levine DA, Soslow RA, Zaloudek C, Shih Ie M and Kurman RJ: Molecular alterations of TP53 are a defining feature of ovarian high-grade serous carcinoma: A re-review of cases lacking TP53 mutations in The Cancer Genome Atlas ovarian study. Int J Gynecol Pathol 35: 48-55, 2016. PMID: 26166714. DOI: 10.1097/PGP.0000000000000207

19 Sakai K, Ukita M, Schmidt J, Wu L, De Velasco MA, Roter A, Jevons L, Nishio K and Mandai M: Clonal composition of human ovarian cancer based on copy number analysis reveals a reciprocal relation with oncogenic mutation status. Cancer Lett 405: 22-28, 2017. PMID: 28734796. DOI: 10.1016/j.canlet. 2017.07.013

$20 \mathrm{Na} \mathrm{K}$ and Kim HS: Clinicopathological characteristics of fallopian tube metastases from primary endometrial, cervical, and nongynecological malignancies: A single institutional experience. Virchows Arch 471: 363-373, 2017. PMID: 28702779. DOI: $10.1007 / \mathrm{s} 00428-017-2186-\mathrm{z}$

$21 \mathrm{Na} \mathrm{K}$ and Kim HS: Clinicopathologic and molecular characteristics of mesonephric adenocarcinoma arising from the uterine body. Am J Surg Pathol 43: 12-25, 2019. PMID: 29189288. DOI: 10.1097/PAS .0000000000000991

$22 \mathrm{Na} \mathrm{K}$, Lee JY, Sung JY, Kim GM, Koo JS and Kim HS: Comparative clinicopathological and cytomorphological analyses of peritoneal carcinomatosis associated with metastatic breast carcinoma and primary peritoneal/ovarian carcinoma in patients with a history of breast carcinoma. Virchows Arch 473: 165-175, 2018. PMID: 29926183. DOI: 10.1007/s00428-018-2390-5

23 Rhead B, Karolchik D, Kuhn RM, Hinrichs AS, Zweig AS, Fujita PA, Diekhans M, Smith KE, Rosenbloom KR, Raney BJ, Pohl A, Pheasant M, Meyer LR, Learned K, Hsu F, HillmanJackson J, Harte RA, Giardine B, Dreszer TR, Clawson H, Barber GP, Haussler D and Kent WJ: The UCSC Genome Browser database: Update 2010. Nucleic Acids Res 38: D613619, 2010. PMID: 19906737. DOI: 10.1093/nar/gkp939

24 Mills RE, Luttig CT, Larkins CE, Beauchamp A, Tsui C, Pittard WS and Devine SE: An initial map of insertion and deletion (INDEL) variation in the human genome. Genome Res 16: 11821190, 2006. PMID: 16902084. DOI: 10.1101/gr.4565806

25 Jang MI, Sung JY, Kim JY and Kim HS: Clinicopathological characteristics of metaplastic papillary tumor of the fallopian tube. Anticancer Res 37: 3693-3701, 2017. PMID: 28668862.

$26 \mathrm{Na} \mathrm{K}$, Kim EK, Jang W and Kim HS: CTNNB1 mutations in ovarian microcystic stromal tumors: Identification of a novel deletion mutation and the use of pyrosequencing to identify reported point mutation. Anticancer Res 37: 3249-3258, 2017. PMID: 28551672. DOI: 10.21873/anticanres.11688

$27 \mathrm{Na} \mathrm{K}$, Park SY and Kim HS: Clinicopathological characteristics of primary ovarian adenomyoma: A single-institutional experience. Anticancer Res 37: 2565-2574, 2017. PMID: 284768 28. DOI: 10.21873 /anticanres. 11600

$28 \mathrm{Na} \mathrm{K}$, Sung JY and Kim HS: Stromal p16 overexpression in adult granulosa cell tumors of the ovary. Anticancer Res 37: 2437-2444, 2017. PMID: 28476811. DOI: 10.21873/anticanres. 11583
$29 \mathrm{Na} \mathrm{K}$, Sung JY and Kim HS: Clinicopathological characteristics of high-grade squamous intraepithelial lesions involving condyloma acuminatum. Anticancer Res 38: 1767-1774, 2018. PMID: 29491115. DOI: 10.21873 /anticanres.12414

30 Li MM, Datto M, Duncavage EJ, Kulkarni S, Lindeman NI, Roy S, Tsimberidou AM, Vnencak-Jones CL, Wolff DJ, Younes A and Nikiforova MN: Standards and guidelines for the interpretation and reporting of sequence variants in cancer: A joint consensus recommendation of the Association for Molecular Pathology, American Society of Clinical Oncology, and College of American Pathologists. J Mol Diagn 19: 4-23, 2017. PMID: 27993330. DOI: 10.1016/j.jmoldx.2016.10.002

31 Khoury MP and Bourdon JC: The isoforms of the p53 protein. Cold Spring Harb Perspect Biol 2: a000927, 2010. PMID: 20300206. DOI: $10.1101 /$ cshperspect.a000927

32 Testa U, Petrucci E, Pasquini L, Castelli G and Pelosi E: Ovarian cancers: Genetic abnormalities, tumor heterogeneity and progression, clonal evolution and cancer stem cells. Medicines 5, 2018. PMID: 29389895. DOI: 10.3390/medicines5010016

33 Soussi T and Wiman KG: TP53: An oncogene in disguise. Cell Death Differ 22: 1239-1249, 2015. PMID: 26024390. DOI: 10.1038/cdd.2015.53

34 Yemelyanova A, Vang R, Kshirsagar M, Lu D, Marks MA, Shih Ie $\mathrm{M}$ and Kurman RJ: Immunohistochemical staining patterns of p53 can serve as a surrogate marker for TP53 mutations in ovarian carcinoma: An immunohistochemical and nucleotide sequencing analysis. Mod Pathol 24: 1248-1253, 2011. PMID: 21552211. DOI: $10.1038 /$ modpathol.2011.85

35 Bartek J, Iggo R, Gannon J and Lane DP: Genetic and immunochemical analysis of mutant p53 in human breast cancer cell lines. Oncogene 5: 893-899, 1990. PMID: 1694291

36 Iggo R, Gatter K, Bartek J, Lane D and Harris AL: Increased expression of mutant forms of $p 53$ oncogene in primary lung cancer. Lancet 335: 675-679, 1990. PMID: 1969059.

37 Brachova P, Mueting SR, Devor EJ and Leslie KK: Oncomorphic TP53 mutations in gynecologic cancers lose the normal protein: Protein interactions with the microRNA microprocessing complex. J Cancer Ther 5: 506-516, 2014. PMID: 25339994. DOI: $10.4236 /$ jct.2014.56058

$38 \mathrm{Gu}$ J, Nie L, Wiederschain D and Yuan ZM: Identification of p53 sequence elements that are required for MDM2-mediated nuclear export. Mol Cell Biol 21: 8533-8546, 2001. PMID: 11713288. DOI: $10.1128 / \mathrm{MCB} .21 .24 .8533-8546.2001$

39 Nie L, Sasaki M and Maki CG: Regulation of p53 nuclear export through sequential changes in conformation and ubiquitination. J Biol Chem 282: 14616-14625, 2007. PMID: 17371868. DOI: 10.1074/jbc.M610515200

40 Xu J, Reumers J, Couceiro JR, De Smet F, Gallardo R, Rudyak S, Cornelis A, Rozenski J, Zwolinska A, Marine JC, Lambrechts D, Suh YA, Rousseau F and Schymkowitz J: Gain of function of mutant p53 by coaggregation with multiple tumor suppressors. Nat Chem Biol 7: 285-295, 2011. PMID: 21445056. DOI: 10.1038/nchembio. 546

41 Goh AM, Coffill CR and Lane DP: The role of mutant p53 in human cancer. J Pathol 223: 116-126, 2011. PMID: 21125670. DOI: $10.1002 /$ path.2784

Received April 10, 2019

Revised April 25, 2019

Accepted April 30, 2019 